\title{
Las relaciones ciencia, tecnología, sociedad y ambiente, para favorecer las actitudes hacia el aprendizaje de la química "El calentamiento global"
}

\author{
Science, technology, society and environment relations for encourage the attitudes \\ towars the learning of chemistry. "Global warming"
}

Laura Milena Cala Cristancho

Profesora en formación. Universidad Pedagógica Nacional. laito cal@hotmail.com

\section{Resumen}

Este trabajo se realizó en el 2007 y 2008, con estudiantes de undécimo del I.E.D. Liceo Femenino Mercedes Nariño, en el espacio de la práctica pedagógica y didáctica I y II. Se dividió en dos etapas, la primera, caracterizar las actitudes de las estudiantes frente al aprendizaje de la química y el diseño de una estrategia de intervención basada en relaciones CTSA para abordar la temática de gases y mejorar los ambientes de aprendizaje reflejados en las actitudes y disposición de las estudiantes, mediante una unidad didáctica basada en el calentamiento global y la contaminación atmosférica; la segunda, la aplicación de la unidad diseñada y la evaluación. Las estudiantes dieron explicación a fenómenos de la temática desde la química e involucrarse a nivel cient́fico, tecnológico, social y ambiental. Finalmente, se dio un cambio de actitud hacia el aprendizaje de la química y en la metodología del trabajo en dase.

\section{Palabras clave}

Actitudes, relaciones CTSA, cambio metodológico, enseñanza, aprendizaje, unidad didáctica

\section{Abstract}

This work was carried out in 2007 and 2008, with eleventh grade students from I.E.D. Liceo Femenino Mercedes Nariño, in the space of teaching practice and teaching I and II. It was divided into two stages, the first for the characterization of the attitudes of students against the learning of chemistry and designing an intervention strategy based on relationships CTSA to address the issue of gas and improve learning environments refiected in attitudes and willingness of the students, through a teaching unit based on global warming and air pollution and the second, the implementation of the design and evaluation unit This unit allowed the students to give explanations of phenomena topic from chemistry and in turn, involve scientific, technological, social and environmental. Finally, there was a change in attitudes towards leaming chemistry and in methods of work in class.

\section{Keywords}

Attitudes, CTSA relations, methodological change, teaching, learning, didactic unit

\section{Introducción}

Uno de los principales problemas del aprendizaje de las ciencias experimentales, como la química, es el de las actitudes desfavorables que poseen las estudiantes frente al aprendizaje, causadas por diversos factores que afectan la disposición y la motivación por aprender ciencia. Los resultados de las investigaciones sobre las actitudes de los adolescentes hacia el aprendizaje de la 
ciencia han mostrado ampliamente cómo éstas son un factor determinante en el proceso de aprendizaje. El desarrollo de estrategias enfocadas en las relaciones CTSA, permite mejorar los ambientes de aprendizaje y romper la monotonía de los modelos tradicionales, a través de relaciones más contextualizadas de ciencia, tecnología, sociedad y ambiente.

A partir de lo observado desde el currículo oculto, en la actualidad las adolescentes tienen otros intereses diferentes al del estudio de las ciencias. Adicionalmente a esto en el I.E.D. Liceo Femenino Mercedes Nariño, desde todas las áreas de formación, se hace poco énfasis en el desarrollo de actitudes hacia el aprendizaje desde enfoques CTSA, que contribuya a una formación de ciudadanas responsables de su conocimiento y de acción de este en su cotidianidad.

\section{Desarrollo}

En esta investigación se planteó la siguiente pregunta: ¿Se pueden mejorar las actitudes hacia el aprendizaje de la química de las alumnas del grado once, de la Institución Educativa Distrital Liceo Femenino Mercedes Nariño, aplicando una estrategia metodológica basada en el enfoque de las relaciones CTSA en la identificación y resolución de problemáticas ambientales?

Esta investigación tuvo como fin mejorar dichas actitudes desfavorables a partir de la resolución de problemáticas ambientales como el calentamiento global, relacionar la química con aspectos de la vida e identificar problemáticas de ambientales que involucren las relaciones CTS y formular soluciones haciendo uso de sus conocimientos.

Para abordar el problema es conveniente revisar brevemente las siguientes cuestiones:

\section{¿Qué se conoce como actitudes?}

La palabra actitud se deriva del latín "aptus" que significa capacidad o adaptación. La actitud es una condición motivadora de cada persona, que determina la forma de pensar y cada uno de los actos que realiza durante toda la vida hacia cosas, personas o actividades. Las actitudes desfavorables hacia el aprendizaje de las ciencias dependen de diversas variables, dentro de las que se pueden distinguir las extemas al aula, las intemas del aula, las relacionadas con el sexo, los textos y medios de comunicación y rasgos de personalidad.

¿Qué significado y relación tiene el enfoque CTSA con la enseñanza y el aprendizaje de la química?

La desarticulación existente entre los contenidos que se enseñan en química y los aspectos de la cotidianidad que viven los estudiantes, constituyen un problema evidente, en la medida que los estudiantes no se interesan por aprender temáticas trabajadas en la clase de ciencias, tal vez al no encontrar en ellas una utilidad apreciable (Rojas y Martínez, 2005).

El enfoque CTSA, se considera en primer lugar, como un campo de estudio e investigación que permite que el estudiante comprenda la relación entre la ciencia con la tecnología y su contenido socio-ambiental (Martínez, Villamil y Peña, 2006); en segundo lugar, como una propuesta educativa innovadora con la finalidad de formar conocimientos y valores que favorezcan la participación ciudadana en aspectos sociales y ambientales.

¿Cuál es la relación del enfoque CTSA en la formación de actitudes hacia las ciencias?

El enfoque CTSA, genera cambios actitudinales en los estudiantes desde ámbitos pedagógicos y didácticos, donde el aprendizaje de los conceptos es más apropiado de acuerdo a su cotidianidad. Una de las metas más aceptadas de este enfoque en la enseñanza de las ciencias experimentales, es que tiene como fin último formar ciudadanos tecnológica y científicamente alfabetizados, 
capaces de tomar decisiones y acciones responsables (Rubba y Wiesenmayer, 1988, citados por Membiela, 1997).

La química en la cotidianidad y la resolución de problemas

La Química es la Ciencia que estudia las propiedades de las diversas sustancias y sus transformaciones. Sin embargo, esta definición no involucra otros temas que esta disciplina abarca, ni la posición central que ocupa entre las ciencias naturales (Zalts, Anita y Pinto, Gabriel, 2003, en Didáctica de la química y vida cotidiana). Por ejemplo, el efecto invernadero, la lluvia ácida, el agujero de ozono, el tratamiento de los residuos urbanos, la comosión, la producción de alimentos,..., son innumerables aspectos y problemáticas cotidianas de la época contemporánea, que se encuentran estrechamente vinculados con la Química y que involucran ejes tecnológicos, sociales y ambientales, que paralelamente otorgan procedimientos y soluciones.

En el campo de la didáctica de las ciencias, la resolución de problemas ha sido estudiada como estrategia para generar cambios conceptuales, metodológicos y actitudinales.

¿Qué es el calentamiento global?

El calentamiento global es una teoría basada en observaciones que muestran un aumento en la temperatura media de la atmósfera terrestre y de los océanos en las últimas décadas. ¿Por qué se produce este calentamiento? El efecto invernadero es una actividad natural que le permite al planeta retener parte de la energía solar (a través de los gases invernadero) que atraviesa la atmósfera, para así mantener una temperatura estable en la Tierra que garantice la subsistencia de las especies que la habitan. El resto de los rayos solares vuelven al espacio. Sin embargo, el incremento de la actividad humana, la quema de combustibles, la producción de carbón, la deforestación, la ganadería, etc, han incrementado la emisión de estos gases invemadero, ocasionando que la atmósfera concentre mayor cantidad de calor y recaliente al Planeta y una serie de consecuencias graves que afectan la normalidad de la tienra y el hombre.

\section{Metodología}

La investigación se dividió en dos etapas. La primera consistió en la caracterización de las actitudes de las estudiantes hacia el aprendizaje de la química a través de cuestionarios y de observaciones diarias, y el diseño de una estrategia de intervención. La segunda consistió en la aplicación de la estrategia: Interacciones CTSA para favorecer las actitudes hacia el aprendizaje de la química, a partir de la resolución de problemas cotidianos: "El calentamiento global'. Esta etapa se dividió en auatro fases, la primera, sobre la determinación de ideas previas de los gases y la determinación de las relaciones CTSA. La segunda fase sobre la contextualización y conocimiento de la temática y la problemática, a través de videos cortos, lecturas, noticias y debates sobre el calentamiento global y los gases invernadero. En tercer lugar, la fase correspondiente a la resolución de problemas basado en el diseño de un biodigestor para reducir la emanación de gas metano a la atmosfera y el estudio del comportamiento de los gases en este sistema. Finalmente, la evaluación para la unidad, el aprendizaje y la actitud.

\section{Conclusiones}

El desarrollo actividades, tales como: video-foros, lecturas, noticias, debates y resolución de problemas, orientadas desde un enfoque CTSA, permitió obtener cambios metodológicos y actitudinales en la enseñanza y aprendizaje de la química, teniendo en cuenta que es importante tanto para el maestro, desarrollar nuevas estrategias en el aula que permitan la participación de sus estudiantes y la generación de actitudes favorables, como para las estudiantes relacionar conceptos químicos involucrados en la temática del calentamiento global para dar explicación a 
fenómenos que suceden en esta problemática y la participación activa en la toma de decisiones como ciudadanas para contribuir a la resolución de un problema ambiental.

Bibliografía

Martínez, L, Villamil, Y. y Peña, D. (2006). Actitudes favorables hacia la química a partir del enfoque Ciencia, Tecnología, Sociedad y Ambiente (CTSA). I Congreso Iberoamericano de Ciencia, Tecnología, Sociedad e Innovación CTS+I.

Martínez, L, Villamil, Y. y Peña, D. (2006). Relaciones Ciencia, Tecnología, Sociedad y Ambiente, a partir de casos simulados. I Congreso Iberoamericano de Ciencia, Tecnología, Sociedad e Innovación CTS+I.

Membiela, P. (1997) Una revisión del movimiento educativo Ciencia-Tecnología-Sociedad. Enseñanza de las ciencias. Revista de investigación y experiencias didácticas. Vol 15 No. 1. 51-57.

Pinto, G. (Editor). (2003) Didáctica de la Química y la vida cotidiana. Sección de Publicaciones de la Escuela Técnica Superior de Ingenieros. Madrid.

Rojas, Á. y Martínez, L (2005). "Estrategia pedagógica y didáctica desde el enfoque ciencia, tecnología, sociedad y ambiente, a partir de las fumigaciones con glifosato". En sala de Lectura, OEI. http://unw.campus-oei.org/salactsi. 2005 